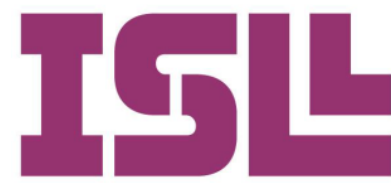

Número 7.

Enero de 2017

\title{
Reading motivation and reading habits of future teachers
}

Sergio Vera Valencia

Dpto. de Psicología

Universidad de Castilla-La Mancha

Pág. 85 a la 96

\section{Abstract:}

According to PISA 2009 results are frequent readers. In addition,

\section{Keywords}

Reading habits, Reading motivation, Preservice teachers, Reading competence, PISA (OCDE, 2010), the Reading literacy of Spanish teenagers is under the international average, and reading motivation and reading habits are highly related to reading performance. And ass research reveals that teachers can be very important for the development of reading pleasure (Gambrell, 1996; Guthrie, 2008) specially if they are reading models (De Neaghel \& Van Keer, 2013), the objective of this study is to explore reading habit and reading motivation in preservice teachers.

To do so, it has been used a reading habit questionnaire for university students (Larrañaga \& Yubero, 2005) and the reading motivation scale develop for PISA 2009 (OCDE, 2010) with 433 Education undergraduates of the University of Castilla-La Mancha.

Results confirm the significant association between reading habits and reading motivation. However, while a high proportion of the participants declare high or very high intrinsic reading motivation, very few differences in reading motivation and reading habit depending on the course were not found.

Implications of the discrepancies found between what future teachers say and do as readers are discussed 



\section{Justificación}

De acuerdo con los resultados de uno de los más influyentes proyectos de evaluación de la competencia lectora, el Programme for International Student Assesment (PISA), España sigue por debajo de la media de la OCDE, ubicándose el 19,6\% de nuestros alumnos de 15 años en el nivel de competencia más bajo (OCDE, 2010).

Unos datos tan alarmantes, que han trascendido el ámbito educativo para convertirse en motivo de preocupación social, a tenor de que estudios longitudinales como los de Bushnik, Telford y Bussiere (2004), Knighton y Bussiere (2006) o Hillman y Thomson (2006), encuentran que los estudiantes en ese nivel tenían muchas probabilidades de no concluir la educación obligatoria, y que según el informe Educación para todos de la UNESCO (2012) el principal motivo por el que nuestro país ostenta el mayor índice de desempleo juvenil de toda la Unión Europea es, precisamente, porque es el país que mayor tasa de fracaso escolar posee.

En este sentido, el informe español de la última edición de PISA centrada en lectura (MECD, 2011), señala que una de las labores más eficaces que puede desarrollar el centro escolar para paliar las diferencias entre clases sociales es promover el interés hacia la lectura. De hecho, en PISA 2000, resultó mayor la correlación entre la competencia lectora y el compromiso con la lectura que con el estatus socio-económico.

En PISA 2009, se observó como en todos los países existía una fuerte asociación entre el rendimiento lector y algunas de las variables del compromiso con la lectura individual, que la prueba concibe como aquellos elementos motivadores y características conductuales de la lectura, entre las que se cuentan la motivación y el hábito lector (OCDE, 2010).

Conscientes de lo dicho, y de que alcanzar un nivel lector satisfactorio es fruto de numerosos años de práctica, León, Escudero y Olmos (2012) sostienen que los bajos resultados obtenidos en PISA tienen su origen en la desmotivación hacia la lectura que los alumnos arrastran de cursos previos.

\subsection{Motivación y hábito lector}

Etimológicamente, motivación es una palabra de origen latino, que viene del verbo "movere", que significa "mover" o "dirigir en una dirección". Aunque el concepto parezca claro, según la revisión teórica de Unrau y Quirkman (2014), existen casi tantas acepciones de motivación como expertos en el área, y no hay consenso en los elementos que componen el constructo o los procesos por los que éstos operan.

En un intento por solventar las dificultades que esta indeterminación acarrea para la investigación sobre motivación a la lectura, Conradi, Jan y McKennan, 2014 proponen una 
definición a partir de la síntesis de su revisión conceptual, según la cual sería "el impulso a leer resultante de un extenso conjunto de creencias sobre actitudes hacia y metas de lectura de un individuo" (p. 25).

Sea como fuere, en PISA 2009 se observa como en todos los países existe una fuerte asociación entre motivación y competencia lectora (OCDE, 2010), y existe evidencia de que la motivación a la lectura es clave no sólo para el rendimiento, sino también para la formación del hábito lector (Schiefele, Schaffner, Moller y Wigfield, 2012).

El diccionario de la Real Academia de la lengua define hábito, en primer lugar como costumbre o práctica adquirida por repetición de un acto; y en segundo, como destreza que se adquiere por el ejercicio repetido. En el caso del hábito lector, será su concepción de hábito como costumbre la que más se acerque a la comúnmente aceptada por la comunidad científica y los medios de comunicación. Así, Cerrillo, Larrañaga y Yubero (2002) lo entienden como una conducta estable que lleva a la persona a leer voluntariamente para ocupar parte de su tiempo de ocio.

Según el informe español de PISA 2009 (MECD, 2011), el tiempo que los jóvenes dedican a la lectura es un factor relevante para su rendimiento lector. Los adolescentes con mejor rendimiento tienden a leer más, mientras que los más retrasados evitan las ocasiones de leer y, por tanto, sus posibilidades de mejora se reducen.

Un trabajo sobre el perfil lector de los españoles entre 1974 y 2010 (BurgosBordonau y Palacios Gómez, 2010), concluyó que las variables sociodemográficas más significativas para caracterizar a un lector en nuestro país son la edad, el sexo, el nivel educativo y la ocupación.

De acuerdo con el último estudio nacional de la FGE (2013), que no distingue entre lectura voluntaria y profesional, la media de libros leídos al año se sitúa en 10,6, y el 88,6\% de los españoles de 14 o más años afirma leer al menos una vez a la semana. Un 79,4\% lee periódicos, un $63 \%$ libros, un 47,6\% revistas, un 13,2\% cómics, y un $46 \%$ webs, foros y blogs al menos una vez al trimestre.

Las mujeres leen más libros $(69 \%)$ y revistas $(58,8 \%)$, mientras los hombres consumen más prensa $(85,5 \%)$, cómics $(17 \%)$ y webs, blogs y foros $(53 \%)$.

En lo que se refiere a la edad, el hábito lector decrece con los años. El tramo en que más se lee es el de 14 a 24 (97,7\%), disminuyendo progresivamente (96,5\% entre los 25 y $34,94,9 \%$ entre los 35 y los $44,92,8 \%$ entre 45 y $54,89,2 \%$ entre 55 y 64 y $81 \%$ en los mayores de 65). 
Tomando en consideración el nivel de estudios, el índice lector aumentará con la cualificación. De esta forma, los que menos leen serán los españoles con estudios primarios (83\%), después los que tienen títulos secundarios $(94,5 \%)$ y son los titulados universitarios los que más lo hacen $(98,4 \%)$.

En cuanto a la ocupación, son los estudiantes $(84,3 \%)$ los que menos leen, seguidos de cerca por los jubilados $(84,1 \%)$, los parados $(94,3 \%)$, las amas de casa $(94,7 \%)$, y los ocupados $(99,6 \%)$.

En suma, que según los datos de la FGEE (2013) hoy en día el perfil del lector habitual en España sería una mujer, joven, universitaria y trabajadora.

Ahora, que si bien los datos sobre hábitos lectores de la población española indican que hoy se lee más que nunca, como concluyen Cerrillo, Larrañaga y Yubero (2007), eso no significa que el número de lectores sea el adecuado para un país desarrollado ni que todos ellos sean lectores competentes, como atestiguan los resultados de PISA 2009.

\subsection{La motivación a la lectura y el hábito lector de los educadores}

La investigación pone de relieve como los maestros pueden ser muy importantes para el desarrollo de la motivación lectora (Gambrell, 1996; Guthrie, 2008), y que para despertar la motivación de sus alumnos los docentes deberían ser modelos lectores, demostrando en clase que valoran la lectura y compartiendo su amor por ella (De Neaghel y Van Keer, 2013).

A pesar de todo, hasta donde se sabe los estudios sobre motivación lectora de los docentes son escasos. Applegate y Applegate $(2004 ; 2014)$ encontraron que menos de la mitad de los futuros maestros de primaria declaraban ser lectores entusiastas, y Nathanson, Pruslow y Levitt (2007) llegaban a similares conclusiones en una muestra de docentes en activo. Algo alarmante a la luz de lo que Applegate y Applegate (2004) denominaron el "efecto Pedro", en referencia a un pasaje bíblico en que un mendigo pedía dinero a San Pedro y éste le respondía: "no se puede dar lo que no se tiene".

De otra parte, según Granado y Puig (2014), no abundan los estudios sobre el maestro como lector, pero los publicados revelan que la lectura no está muy presente en la vida de los docentes. Pocos maestros leen con frecuencia lecturas personales y/o profesionales (Applegate y Applegate, 2004; Benevides y Peterson, 2010; Cremin, Bearne, Mottram y Goodwin, 2008; Tenti-Fanfani, 2005), y apenas utilizan las bibliotecas (Cremin et al., 2008; Marchesi y Miret, 2005; Romero, 2007).

En cuanto a los futuros maestros españoles, Granado y Puig (2014) hallaron que el $65 \%$ de los encuestados leían menos que la media nacional y que el 88,6\% compraba menos libros que el promedio nacional. Sólo el 3\% leía más de 12 libros al año y el $60 \%$ dedicaba

ISL, vol. 7, 2017, págs. 85-96 ISSN: $2340-8685$
Vera Valencia, S. (2017): Reading motivation and reading habits of future teachers, Investigaciones Sobre Lectura, 7, 85-96. 
entre cinco y siete horas semanales a la lectura. Unas cifras que las investigadoras consideraron muy bajas, especialmente cuando la edad de la mayoría de los participantes osciló entre 14 y 24 años, que como ya se ha visto, es el tramo en que más se lee en España.

Así pues, a la luz de estos hallazgos, el objetivo del presente trabajo será analizar la motivación y el hábito lector de futuros educadores, y nuestras hipótesis serán:

1. La gran mayoría de futuros educadores no son lectores frecuentes ni intrínsecamente motivados.

2. Existe una asociación significativa entre motivación y hábito lector en los futuros educadores.

3. Se aprecian diferencias significativas en la motivación y hábito lector de los estudiantes de Educación en función del curso.

\section{Método}

\subsection{Participantes}

La muestra se compuso de 433 estudiantes (24\% chicos, $76 \%$ chicas), de edades comprendidas entre 18 y 43 años $(M=21,45$, D.T.= 3,63), que cursaban los grados de Primaria e Infantil en Facultades de Educación de la Universidad de Castilla- la Mancha $\left(28,2 \% 1^{\circ}\right.$ curso, $40,2 \% 2^{\circ}$ curso, y $31,6 \% 3^{\circ}$ curso $)$.

Su selección ha sido incidental, aunque todos los participantes decidieron colaborar libre y voluntariamente en la investigación, sin percibir recompensa alguna por ello.

\subsection{Instrumentos}

Para realizar este estudio de corte cuantitativo, se ha empleado un cuestionario que evaluaba:

- Variables sociodemográficas (sexo, edad y curso).

- El Hábito lector que, de acuerdo con el cuestionario validado con universitarios españoles por Larrañaga y Yubero (2005) evaluamos cruzando el comportamiento lector y el número de libros leídos durante el último año en su tiempo libre, diferenciando cuatro grupos de lectores: no lectores, que nunca o casi nunca leen, entre 0 y 2 libros al año; lectores ocasionales, que leen alguna vez al trimestre o al mes, entre 3 y 10 libros al año; falsos lectores, que en una variable se muestran como lectores ocasionales y en otra como no lectores; y lectores frecuentes, que leen casi todos los días o más de una vez a la semana, más de 10 libros al año.

- Motivación a la lectura, empleando para ello el cuestionario de "Disfrute de la lectura" de PISA 2009 (OCDE, 2010), una escala unifactorial de 11 
ítems y cuatro puntos, desde completamente de acuerdo a completamente en desacuerdo, que mide la motivación intrínseca hacia la lectura, y que ha mostrado alta consistencia interna (alfa=.92).

\subsection{Procedimiento}

Según la clasificación efectuada por Montero y León (2007), la presente investigación es un estudio descriptivo transversal mediante encuestas.

La recogida de datos fue llevada a cabo entre abril y mayo de 2015 por el investigador y dos alumnos debidamente adiestrados para ello. Las pruebas fueron administradas a cada grupo natural de forma colectiva dentro del aula, incidiendo en la confidencialidad de los datos y la importancia de la sinceridad, para reducir la deseabilidad social.

\subsection{Análisis de datos}

Primero, se ha comprobado la fiabilidad y validez del cuestionario de motivación mediante el cálculo del Alfa de Cronbach y el Análisis Factorial Confirmatorio a través de la extracción de componentes principales con rotación Varimax.

A continuación, se calcularon las frecuencias de la motivación y el hábito lector, una vez cruzados el comportamiento lector y el número de libros leídos.

En tercer lugar, se analizó el grado de asociación entre motivación y hábito lector mediante una prueba chi cuadrado.

Por último, se efectuó una ANOVA a fin de comparar las puntuaciones en hábito y motivación en función del curso

Todos los cálculos se realizaron con el paquete estadístico SPSS 21.

\subsection{Resultados}

2.5.1. Análisis factorial y consistencia interna del cuestionario de motivación a la lectura

Tras invertir los cinco ítems con sentido negativo de la Escala de motivación a la lectura $(1,4,6,8$ y 9) el análisis factorial muestra la estructura unifactorial esperada, que explica el 48,676\% de la variancia y demuestra una elevada consistencia interna (.889).

ISL, vol. 7, 2017, págs. 85-96 ISSN: $2340-8685$
Vera Valencia, S. (2017): Reading motivation and reading habits of future teachers, Investigaciones Sobre Lectura, 7, 85-96. 


\begin{tabular}{l|l|c}
1 & Solo leo por obligación &, 766 \\
\hline 2 & Leer es una de mis aficiones favoritas &, 844 \\
\hline 3 & Me gusta hablar sobre libros con otras personas &, 800 \\
\hline 4 & Me cuesta terminar los libros &, 460 \\
\hline 5 & Me encanta que me regalen libros &, 787 \\
\hline 6 & Para mí, leer es una pérdida de tiempo &, 574 \\
\hline 7 & Disfruto yendo a librerías y bibliotecas &, 669 \\
\hline 8 & Solo leo para obtener la información que necesito &, 789 \\
\hline 9 & No puedo sentarme tranquilo y leer durante más de unos pocos minutos &, 631 \\
\hline 10 & Me gusta dar mi opinión sobre los libros que he leído &, 545 \\
\hline 11 & Me gusta intercambiar libros con mis amigos &, 700
\end{tabular}

De acuerdo con nuestros datos, 119 (27,5\%) de nuestros futuros educadores leen por placer al menos una vez al día o una vez a la semana, $140(32,3 \%)$ nunca o casi nunca lee y $174(40,2 \%)$. asegura hacerlo mensual o trimestralmente.

Sin embargo, 215 participantes $(49,5 \%)$ declaran haber leído por placer entre $0 \mathrm{y}$ 2 libros durante el último año, 188 (43\%) entre 3 y 10, y sólo los 30 restantes $(6,9 \%)$ lee más de 10 libros, y podrían ser considerados lectores frecuentes.

Por ello, al entrecruzar ambas variables con objeto de calcular el hábito lector, siguiendo el procedimiento descrito por Larrañaga y Yubero (2005), 126 encuestados $(29,1 \%)$ son categorizados como no lectores, 89 (20,6 \%) son falsos lectores, $195(45 \%)$ son lectores ocasionales y únicamente $23(5,3 \%)$ lectores frecuentes.

En lo que respecta a la motivación a la lectura, como no se tiene constancia de que el instrumento seleccionado haya sido utilizado de forma aislada y no se dispone de orientaciones que permitan valorar sus resultados, se ha optado por elaborar nuestra propia clasificación.

Así, una vez sumadas las puntuaciones de los 11 ítems, se han diferenciado cuatro niveles de motivación a la lectura: muy baja, para aquellos casos en que las puntuaciones oscilen entre 11 y 22; baja, cuando se sitúen entre 23 y 33,; alta, si lo hacen entre 34 y 44; y muy alta, si es entre 45 y 55.

Pues bien, de acuerdo con esta clasificación, 26 de los participantes (6\%) evidencian una motivación lectora muy baja, $140(32,3 \%)$ baja, $168(43,8 \%)$ alta y 85 $(19,6 \%)$ muy alta.

\subsubsection{Asociación entre la Motivación y el Hábito lector}

Encontramos Asociación significativa entre Hábito y Motivación a la lectura (X $=208 \mathrm{P}<0.01$ ). A este respecto, como puede apreciarse en la tabla 2, llama la atención 
que existan no lectores que declaren tener una motivación alta, falsos lectores con motivación alta e incluso muy alta, y mayor número de lectores ocasionales que de lectores frecuentes con motivación muy alta.

Motivación a la lectura

\begin{tabular}{l|l|l|l|l|l}
\multicolumn{1}{c|}{} & Muy Baja & Baja & Alta & Muy Alta \\
\multirow{3}{*}{ Hábito } & No lector & $21(5 \%)$ & $79(18.9 \%)$ & $21(5 \%)$ & $0(0 \%)$ \\
& Falso lector & $3(0.7 \%)$ & $32(7.6 \%)$ & $45(10.7 \%)$ & $5(1.2 \%)$ \\
& Lector ocasional & $2(0.5 \%)$ & $29(6.9 \%)$ & $97(23.2 \%)$ & $63(1.5 \%)$ \\
& Lector frecuente & $0(0 \%)$ & $0(0 \%)$ & $5(1.2 \%)$ & $17(4.1 \%)$
\end{tabular}

Tabla 2. Asociación entre Hábito lector y Motivación a la lectura

\section{Diferencia por curso en las variables de estudio}

La ANOVA no arroja diferencias significativas en función del curso en Motivación a la lectura $(\mathrm{F}=1,929 \mathrm{P}=0,147)$ ni en Hábito lector $(\mathrm{F}=1,041 \mathrm{P}=0,354)$

\section{Discusión}

El propósito de este trabajo era explorar la motivación y hábito lector en futuros educadores.

Como cabía esperar, un porcentaje muy bajo de los encuestados $(5,9 \%)$ podrían ser considerados lectores frecuentes. No en vano, aunque un $27,5 \%$ aseguraron leer por placer al menos una vez a la semana, sólo un 6,9\% han leído más de 10 libros por placer al año. Datos incongruentes, pero que siguen el mismo patrón que hallaran Granado y Puig (2014). Y es que, si bien el 60\% decía leer varias horas a la semana, sólo un 3, $9 \%$ de sus futuros docentes leía más de 12 libros al año. Inconsistencias que Yubero-Jiménez y Larrañaga-Rubio (en prensa) descubrieron también en una muestra representativa de universitarios españoles y portugueses, y que los investigadores achacan a la deseabilidad social o el autoengaño, es decir, a que los estudiantes de enseñanza superior, y más en el caso que nos ocupa, son conscientes de que deberían leer, y por eso falsean sus respuestas para ofrecer una buena imagen ante los demás o ante sí mismos, para no sentirse descategorizados como universitarios y preservar de esta forma su autoestima.

Algo que también explicaría como, a pesar de su bajo índice lector, el porcentaje de futuros educadores que dicen estar intrínsecamente motivados por la lectura supere notablemente los de Applegate y Applegate (2004; 2014) y Nathanson et al. (2007), con un $43,8 \%$ de participantes manifestando alta motivación lectora y un $19,6 \%$ muy alta, cuando los estudios previos encontraban que menos de la mitad eran lectores entusiastas.

En esta misma línea, se aprecia la asociación significativa entre hábito lector y motivación intrínseca postulada por la revisión de Schiefele et al. (2012), pero también que numerosos participantes manifiestan más motivación que hábito. Así, encontramos que un $5 \%$ son no lectores y un $10,7 \%$ falsos lectores que declaran tener una alta 
motivación por la lectura, y que hay el triple de lectores ocasionales (15\%) que de lectores frecuentes $(4,1 \%)$ con motivación muy alta. Una discrepancia que podría deberse, una vez más, a que los estudiantes son conscientes de que, como educadores en formación, deberían encontrar placer en la lectura, pero que a tenor de su escaso índice lector, en realidad no lo hacen.

Por último, no se ha encontrado la diferencia prevista en función del curso, a la luz de los resultados de un estudio piloto sobre el hábito lector que efectuamos con 113 futuros educadores (Vera, 2014). Un hallazgo que invita a la reflexión, pues sugiere, por una parte, que gran número de los estudiantes que ingresan a la titulación no muestran interés por la lectura, y por otra que los esfuerzos realizados desde la Universidad para fomentarla parecen ser insuficientes.

Sea como fuere, lo indudable es que si los maestros juegan un papel tan relevante en la animación a la lectura como consideran algunos expertos (Gambrell, 1996; Guthrie, 2008), y si para conseguirlo deben ser modelos lectores (De Neaghel y Van Keer, 2013), nuestros participantes son un claro ejemplo del llamado efecto Pedro (Applegate y Applegate, 2004; 2014), por lo que será muy difícil que los futuros educadores logren contagiar una pasión por la lectura que ellos mismos no sienten.

Por ello, tal y como defienden Granado y Puig (2014), será necesario promover más actividades de animación a la lectura entre los futuros encargados de llevarlas a cabo en las escuelas, si queremos que sus alumnos lleguen a ser lectores frecuentes y, vista la estrecha relación que los estudios como PISA ponen entre hábito y rendimiento, también competentes.

Con todo, cabe ser cautelosos en la generalización de estas conclusiones, puesto que además de la deseabilidad social ya mencionada, la principal limitación del presente estudio es la falta de aleatoriedad y tamaño muestral, que impiden que los resultados sean representativos para la población española de futuros educadores.

\section{Bibliografía}

Applegate, A. J., y Applegate, M. D. (2004). The Peter Effect: Reading habits and attitudes of preservice teachers. The Reading Teacher, 57, 554-563

Applegate, A. J., Applegate, M. D., Mercantini, M. E., McGeehan, C.M., Cob, J.B, Deboy, J.R., Madia, V.B.y Lewinsky, K. E. (2014). The Peter effect revisited: reading habits and attitudes of college students. Literary research and instruction, 53 (3), 188-204.

Benevides, T., y Peterson, S. S. (2010). Literacy attitudes, habits and achievements of future teachers. Journal of Education for Teaching, 36(3), 291-302. 
Burgos-Bordonau, E., y Palacios-Gómez, J.L. (2010) El perfil sociodemográfico del lector de libros en España (1975-2010). Revista general de información y documentación, 20(1), 325-341.

Bushnik, T., Telford, L. B. y Bussiére, P. (2004). In and out of high school: First results from the second cycle of the Youth in Transition Survey, 2002. Statistics Canada Education, skills and learning research papers, 81-595-MIE-No. 014

Cerrillo, P.C., Larrañaga, E. y Yubero, S. (2002) Libros, lectores y mediadores. Cuenca: Servicio de publicaciones de la Universidad de Castilla-La Mancha.

Cerrillo, P.C., Yubero, S. y Larrañaga, E. (2007) Libros, lectores y mediadores. En: P.C. Cerrillo y S. Yubero (coords.) La formación de mediadores para la promoción de la lectura (pp.229-236).

Conradi, K, Jang, B.G. y McKenna, M.C. (2014) Motivation terminology in Reading research: a conceptual review. Educational Psychology Review, 26(1), 129-164.

De Naeghel, J., y Van Keer, H. (2013). The relation of student and class-level characteristics to primary school students' autonomous reading motivation: $A$ multilevel approach. Journal of Research in Reading, 36, 351-370.

Cremin, T., Beame, E., Mottram, M, y Goodwin, P. (2008): Primary teachers as readers. English in Education, 42, 8-23.

Federación de Gremios de Editores de España (FGEE) (2013). Hábitos de lectura y compra de libros en España, 2012. FGEE y Ministerio de Cultura. Recuperado el 29 de octubre de 2015, de:

http://www.federacioneditores.Org/0 Resources/Documentos/130207NPR-FGEEBarometroHabitosdeLectura2012.pdf

Gambrell, L. B. (1996). Creating classroom cultures that foster reading motivation. The Reading Teacher, 50, 14-25.

Granado, C. y Puig, M. (2014). Qué leen los futuros maestros y maestras. Un estudio del docente como sujeto lector a través de los títulos de los libros que evocan. OCNOS, 11, 93-112

Guthrie, J. T. (Ed.). (2008). Engaging adolescents in reading. Thousand Oaks, CA: Corwin Press.

Hillman, K. y Thomson, K. (2006). Pathways from PISA: LSAY and the 2003 PISA Sample Two Years On. Melbourne: ACER

ISL, vol. 7, 2017, págs. 85-96 ISSN: $2340-8685$
Vera Valencia, S. (2017): Reading motivation and reading habits of future teachers, Investigaciones Sobre Lectura, 7, 85-96. 
Knighton, T. y Bussiere, P. (2006). Educational Outcomes at Age 19 Associated with Reading Ability at Age 15. Statistics Canada Education, skills and learning research papers, 81-595-MIE-N 043.

Larrañaga, E. y Yubero, S. (2005). El hábito lector como actitud. El origen de la categoría de 'falsos lectores'. OCNOS, 1, 43-60 León, J.A., Escudero, I. y Olmos, R. (2012). Evaluación de la comprensión lectora. Madrid: Tea Ediciones

Marchesi, A., y Miret, I. (2005). Las bibliotecas escolares en España, 2005. Madrid: Fundación Germán Sánchez Ruipérez

Ministerio de Educación (2011). PISA ERA 2009. Programa para la Evaluación Internacional de los Alumnos. Informe español. Madrid: Ministerio de Educación.

Montero, I. y León, O. (2007). A guide for naming research studies in Psychology. International Journal of Clinical and Health Psychology, 7 (3), 847-862.

Nathanson, S., Pruslow, J., y Levitt, R. (2008). The reading habits and literacy attitudes of inservice and prospective teachers: results of a questionnaire survey. Journal of Teacher Education, 59, 313-321.

Organization for Economic Cooperation Development [OECD], (2010). PISA 2009 results: Learning to learn: Student engagement, strategies and practices (Volume III). Paris: OECD Publishing.

Romero, F. (2007). Utopías lectoras y promoción de la lectura en la escuela. Anuario sobre el libro infantil y juvenil 2007 (pp.81-114). Madrid: Ediciones SM.

Schiefele, U., Schaffner, E., Moller, L, y Wigfield, A. (2012). Dimensions of reading motivation and their relation to reading behavior and competence. Reading Research Quarterly, 47, 427- 463.

Tenti-Fanfani, E. (2005). La condición docente. Análisis comparado de la Argentina, Brasil, Perú y Uruguay. Buenos Aires: Siglo XXI.

UNESCO (2012). Educación para todos. París: UNESCO

Unrau, N. J. y Quirk, M. (2014) Reading Motivation and Reading Engagement: Clarifying Commingled Conceptions. Reading Psychology, 35(3), 260-284.

Vera, S. (Junio de 2014). Creencias en torno a la lectura. Enseñar a comprender. Simposio presentado en II Congreso Internacional de Ciencias de la Educación y del Desarrollo. Congreso celebrado en Granada, España. 
Yubero-Jiménez, S. y Larrañaga-Rubio. E. (en prensa) Lectura y Universidad: hábitos lectores de los estudiantes universitarios de España y Portugal. El profesional de la información, 24(6).

ISL, vol. 7, 2017, págs. 85-96 ISSN: $2340-8685$
Vera Valencia, S. (2017): Reading motivation and reading habits of future teachers, Investigaciones Sobre Lectura, 7, 85-96. 